\title{
BMJ Open Association of socioeconomic status with financial burden of disease among elderly patients with cardiovascular disease: evidence from the China Health and Retirement Longitudinal Survey
}

Chunyan Li, ${ }^{1,2}$ Belinda-Rose Young, ${ }^{2}$ Weiyan Jian ${ }^{1}$

To cite: Li C, Young B-R, Jian W. Association of socioeconomic status with financial burden of disease among elderly patients with cardiovascular disease: evidence from the China Health and Retirement Longitudinal Survey. BMJ Open 2018;8:e018703. doi:10.1136/ bmjopen-2017-018703

- Prepublication history and additional material for this paper are available online. To view these files, please visit the journal online (http://dx.doi. org/10.1136/bmjopen-2017018703).

Received 15 July 2017 Revised 30 January 2018 Accepted 28 February 2018

Check for updates

${ }^{1}$ Department of Health Policy and Management, School of Public Health, Peking University, Beijing, China

${ }^{2}$ Department of Health Behavior, The Gillings School of Global Public Health, The University of North Carolina at Chapel Hill, Chapel Hill, North Carolina, USA

Correspondence to

Dr Weiyan Jian;

jianweiyan@bjmu.edu.cn

\section{ABSTRACT}

Objectives The prevalence of cardiovascular diseases (CVD) within low-income and middle-income countries has reached epidemic proportions. However, the association between outof-pocket (OOP) payment and socioeconomic status (SES) of patients with CVD is not well studied. We aimed to understand the financial burden among Chinese middle-aged and older patients with CVD, and whether there was an association with SES.

Settings A nationally representative survey-The China Health and Retirement Longitudinal Survey (CHARLS)—was conducted in 28 provinces of mainland China in 2011 and 2013.

Participants Of the over 18000 CHARLS respondents, eligible participants were those aged 45 years and over who had been previously diagnosed with CVD.

Outcome measures Financial burden was measured by individual 00P payment and household catastrophic health expenditure (CHE) occurrence (ie, the annual household health expenditure was $40 \%$ or more of the total non-food household expenditure). Multilevel regression models were used to explore the association between financial burden and SES.

Results Among CHARLS respondents, CVD prevalence increased from $14.7 \%$ in 2011 to $16.6 \%$ in 2013. Average annual CVD 00P payment increased from 5000 RMB (770 USD) to 6120 RMB (970 USD). Furthermore, CHE occurrence increased from $44.2 \%$ to $48.1 \%$. Patients spent almost twice on outpatient as on inpatient services. Two of the three SES indicators (total household expenditure, occupation type) were found to be associated with CVD 0OP payment amount, and the likelihood of CHE. Unemployed patients had a higher likelihood of CHE compared with agricultural workers. Rural-urban difference was associated with the likelihood of $\mathrm{CHE}$ in 2011 alone.

Conclusion The Chinese health system should use this health expenditure pattern among patients with CVD to create more equitable health insurance schemes that financially balance between outpatient and inpatient care, and provide better financial risk protection to patients with IOW SES.
Strengths and limitations of this study

- Use nationally representative household survey data to estimate health expenditure among patients with cardiovascular disease (CVD).

- Apply multilevel regression model to explore how both individual and contextual socioeconomic features were associated with financial burden among patients with CVD.

- Cost estimation was based on the cost for last medical visit among patients whose reason for their last outpatient/inpatient visit was CVD, which reduces estimation bias resulting from co-occurrence of other non-communicable diseases.

- The database (China Health and Retirement Longitudinal Survey) is a self-reported survey that might result in recalling bias.

\section{INTRODUCTION}

WHO reports that cardiovascular diseases (CVD) (eg, coronary heart disease and cerebrovascular disease) account for $31 \%$ of deaths globally. While CVD mortality rates are globally pervasive, $75 \%$ of CVD deaths occur in low-income and middle-income countries. ${ }^{1}$ The response to this global epidemic has been to better study the risk factors associated with CVD incidence. ${ }^{2}$ However, of equal importance is the need to understand healthcare costs associated with CVD after diagnosis, and the financial burden that it places on a household. Since CVD is a chronic disease, there is a consistent demand for healthcare services and medication among those affected.

The concept of 'new public health' attributes individual's health and their health behaviours partly to the upstream causes of poor health. ${ }^{3}$ These include contextual variables such as physical features of the environment, service availability and sociocultural features within a neighbourhood, which significantly influence 
medical cost. ${ }^{4}$ Persons living in the aforementioned disadvantaged conditions often face higher risk of negative health outcomes, ${ }^{5}$ but have lower financial capacity to pay for adequate healthcare services. In other words, patients with lower socioeconomic status (SES) may spend less money purchasing health services ${ }^{6}{ }^{6}$ but have a higher risk of catastrophic health expenditure (CHE).

According to the 2016 National Report on Cardiovascular Diseases, one in five Chinese adults live with a CVD (including cerebrovascular disease, coronary heart disease, arrhythmia, heart failure, pulmonary arterial hypertension, cardiovascular surgery and chronic kidney disease), and the annual inpatient care cost has risen at a steady rate of $30 \%$ since 2004. ${ }^{7}$ Researchers with an interest in Chinese health policies may be aware of the ongoing, extensive health reform in the country since 2009, which aims to expand universal health coverage and to provide a basic public health package to all citizens. ${ }^{8}$ For instance, the Chinese government now supports basic medical insurance for over $95 \%$ of the population and, as such, service utilisation has more than doubled. ${ }^{9}$ However, health outcomes among the Chinese population have not improved commensurately with the government's effort. One possible reason for this could be that the insurance system is organised into multiple benefit packages that differ by one's employment status and ID registration type. Briefly, at the time of birth Chinese residents may register for an urban ID or a rural ID, mostly based on the family's location. The ID type together with employment status directly impacts their ability to receive various healthcare benefit packages. For example, urban employees are enrolled in the Urban Employee Basic Medical Insurance, whose funding comes from individuals and employers (monthly premium). Urban unemployed residents are enrolled in Urban Resident Basic Medical Insurance (URBMI); and rural residents, regardless of employment status, are enrolled in the New Rural Cooperative Medical Insurance (NRCMI). Those enrolled in either the URBMI or NRCMI individually pay an annual premium and receive some subsidisation by the government. ${ }^{10}$

In addition to basic medical insurance schemes, patients from low-income households and/or who have severe physical conditions may be protected by special medical aid packages, but it often requires that the patient has knowledge of how and when to apply for it, and that they possess the health literacy level to finish the complex application form within their local social insurance department. The inclusion criteria for the special medical aid packages varies across cities. Such a differential design in healthcare benefits has at times resulted in social inequality issues - with higher SES patients typically enjoying better insurance schemes (eg, a higher reimbursement rate, more kinds of covered medical care and medicines) and higher rates of service utilisation. ${ }^{11} 12$ Chinese policymakers have acknowledged the unequal nature of the medical insurance design ${ }^{11}$ and are actively trying to decrease inequalities through investing money in rural health systems and providing supplemental medical aid for patients from poor households. However, the ongoing health reform is not as cost-effective as it could be due to existing inefficiencies in the healthcare system, and scarcity and maldistribution of qualified health workforce across the country. ${ }^{13}$ The healthcare system's disproportionate emphasis on reimbursing inpatient services while not addressing the expensive outpatient services for those suffering with for chronic diseases contribute to the government's poor performance in actually reducing financial burden on patients. ${ }^{14}$ One study based in the Shandong province showed that the NRCMI for rural residents fails to prevent poor households from CHE (defined as the annual household health expenditure is $40 \%$ or more of household's capacity to pay).$^{15}$ Further evidence shows that the NRCMI only reduced the incidence of CHE among the richer households from 2009 to 2012. ${ }^{15}$

Prior research has shown that the financial burden associated with CVD is disproportionate across patient demographics (eg, gender, age). ${ }^{16} 17$ While these two particular studies suggest that SES indicators (income, employment and education) are associated with OOP payment among patients in low-income and middle-income countries, ${ }^{16}{ }^{18}$ we sought to explore whether there was evidence of association within Chinese patients with CVD specifically, and if SES is associated with both individual OOP payment and household CHE. Findings from this provide critical evidence that will enable Chinese policymakers to develop population-appropriate and pro-poor insurance schemes, helping to achieve a more effective and equitable health system. Additionally, evidence from this study contributes to the global body of health systems reform research and is useful in addressing WHO's call for future cross-country comparative research. ${ }^{19}$

\section{METHODS}

\section{Database}

The rationale and methodology of China Health and Longitudinal Retirement Survey (CHARLS) has been reported previously. ${ }^{20}$ Briefly, CHARLS is a nationally representative, longitudinal household survey that examines the social, financial and health circumstances of citizens. Based on a probability proportionate to size sampling strategy, CHARLS covers 450 communities in 150 counties from 28 of the 32 provinces in the mainland China. Households are randomly selected from maps and listings within each rural or urban community. Only residents aged 45 years or older and their spouses (if any, and of any age) were interviewed at baseline and after a 2-year time period. There were a total number of 17708 respondents from 10253 households interviewed in 2011, of which 15775 respondents from 9195 households were successfully followed-up in 2013 (89.08\% retention rate). Another 2841 respondents from 1706 households were recruited in 2013 to the cohort in order to acquire a similar sample size. ${ }^{20}$

\section{Participants}

Eligible participants were residents within the 450 aforementioned communities and their spouses (if 
applicable) that participated in CHARLS. Specifically, we applied two approaches to select patients with CVD from all CHARLS participants: (1) when asked if they had been diagnosed with having a chronic disease by a doctor, respondents who reported being diagnosed with a 'heart attack, coronary heart disease, angina, congestive heart failure or other heart problems' or 'stroke' by a doctor were identified as patients with CVD; (2) respondents who reported one of those diseases as the reason for their last outpatient visit or inpatient hospitalisation, but not necessarily meeting the first inclusion criteria (ie, self-reported as patients with CVD being diagnosed by doctors), were also identified as patients with CVD in our analyses. A total of 2568 respondents in $2011(14.5 \%)$ and 3056 respondents in 2013 (16.4\%) met both our two-step inclusion criteria. In total, 1775 patients out of the 2568 patients $(69.1 \%)$ enrolled in 2011 were retained in 2013.

\section{Measures}

OOP payment and CHE

Health expenditure data contained in the CHARLS questionnaire measures both the direct and indirect costs associated with CVD. Direct costs included OOP payment for self-medication (such as buying over-the-counter medicines, taking home-made herbal medications during the past month prior to the survey) and inpatient and outpatient services (from the last clinic/hospital visit). While indirect costs included OOP spending by the patient on transportation to the outpatient visit, or by the patients and families on transportation, accommodation, food and hiring care workers during inpatient hospitalisation. This study reported direct and indirect spending by outpatient/inpatient visits separately, and used the total annual OOP expenditure as the dependent variable in regression models. It should be noted that in consideration of the potential co-occurrence of other non-communicable diseases (NCDs) among patients with CVD that might increase individual health expenditure, we only included patients whose reason for their last outpatient/inpatient visit was CVD in our cost estimation. Hence, the estimated annual cost was based on the self-reported cost for the last treatment of patients with CVD.

Individual health expenditure in this study was calculated for each patient with CVD, through the following formulas: (1) annual self-medication cost (self-reported self-medication cost during the past month $\times 12$ ); (2) annual direct/indirect outpatient cost (self-reported direct/indirect cost of last outpatient visit $\times$ outpatient visit times during the past month $\times 12$ ) and (3) annual direct/ indirect inpatient cost (self-reported direct/indirect cost of last inpatient visitxinpatienthospitalisation times during the past year). The occurrence of CHE was measured at household level. Previous research has recommended that CHE be defined as $40 \%$ or more of the total non-food household expenditure allocated towards the annual household health expenditure. ${ }^{21} 22$ We used this definition to create a dichotomous variable in order to indicate the presence of CHE within each household ('1=yes' $(\geq 40 \%)$ and ' $0=$ not' $^{\prime}(<40 \%)$ ).

\section{SES indicators}

Individual-level SES was measured by three indicators: education level, occupation type and household expenditure. Previous studies have shown that individual education level might be negatively associated with frequency of health services utilisation (because they are more likely to live healthier lifestyles), ${ }^{23}$ but positively associated with willingness to pay for health services if in need. ${ }^{24}$ Based on respondents' answers, we categorised educational attainment into four levels: primary school, middle school, high school and vocational school and above. Individual occupation at the time of survey is also measured and categorised into five groups: agricultural work (engaged in agricultural work for at least 10 days in the past year, and not self-identified as employed by others, self-employed, retired or unemployed), employed, self-employed, retired (including receded, ie, people who quit their job before retiring age while enjoying the same benefits as normal retirement) and unemployed. Those categorised as unemployed also included urban residents who self-identified as unemployed, and rural residents who did not engage in agricultural work for at least 10 days. As described earlier (in China), a resident's ability to enrol within a particular basic medical insurance scheme depends on his/her employment status and rural/urban residence, thus occupation could play a role in healthcare-seeking behaviour due to medical insurance restrictions.

We used total household expenditure over the past 12 months as the third indicator of SES. We chose to use total household expenditure over household income because past studies have intimated that the ratio of health payments to income might not be responsive to catastrophic expenditure when a household finances healthcare costs from their personal savings. ${ }^{21}$ In CHARLS, household expenditure is measured by a series of factors, such as food expenditure, durable goods purchase, utility bills, communication and transportation cost. Interviewees were asked to report the cost estimate for a specific factor in accordance with the likely frequency of cost. For example, during the past week (eg, food expenditure), month (eg, communication and transportation cost) or year (eg, furniture purchase). It should be noted that when defining the occurrence of CHE, we used household annual non-food expenditure (ie, we do not include food expenditure in the denominator). ${ }^{21}$ To reduce the influence on household economic status from the variation of economy development across different cities, we adopted a relative approach to present one's household annual expenditure level. First, we divided the exact amount of one household annual expenditure by the median level in the city where the household resides, and then grouped all the quotients into five equal partitions (from the lowest $20 \%$ to the highest $20 \%$ ). In addition to the aforementioned three indicators, the rural-urban difference of the patient's 
residency region was also treated as an important SES indicator in this study.

\section{Control variables}

There were four individual-level and five community-level control variables. Individual-level control variables for patients with CVD included: (1) age (45-54, 55-64, 65-74, 75 and older); (2) sex; marital status (living with a spouse or partner, not living with a spouse or partner) and (3) health status. Health status was indicated by the number of co-occurring NCDs (eg, diabetes, dyslipidemia, cancer or malignant tumour, hypertension, chronic lung diseases, kidney diseases, liver disease, stomach or other digestive disease, psychiatric problems, memory-related disease, arthritis or rheumatism, asthma) to CVD (none, one to two co-occurrences, more than three co-occurrences).

Community-level control variables included: (1) population size $(<1500,1500-7000$ and $>7000)$; (2) per capita annual net income; (3) regional location (east/middle/ west part of China); (4) availability of healthcare services and (5) availability of transportation services. The latter two factors were included in our model for potential confounding effects. Here, we used the number of public bus routes $($ none $=$ not good, $1-2$ routes $=$ good, $\geq 3$ routes=verygood) to indicate the accessibility of transportation service, and the number and type of healthcare facilities (none, only primary care centres and having secondary or tertiary hospitals) within the community to indicate the availability of healthcare services at the community level. All information at the community level was directly collected from local officials through a constructed questionnaire.

\section{Statistical analysis}

All statistical analyses were conducted using data from 2011 and 2013 separately. We reported the prevalence of CVD by SES groups first, and then examined the statistical differences by groups with the $\mathrm{X}^{2}$ tests. We used a multilevel linear regression analysis (ie, a two-level random-intercept model with individuals at the first level and communities at the second level) to study the association of individual SES characteristics with the annual OOP payment, among patients with CVD. To normalise the distribution of the error items, the numeric value of OOP payment was log-transformed in the regression model. Subsequently, another two-level random-intercept model using logistic regression was performed to analyse the odds of CHE occurring within family of a patient with CVD, compared with the first group of each SES indicator. In all regression models, individual-level variables included SES indicators (education, occupation and household living expenditure), age, sex, marital status and NCD co-occurrence, as well as community-level characteristics including rural-urban difference, regional location, population size, transportation, number of health facilities and per capita annual net income. All data cleaning and statistical analyses were done using Stata
V.14.1 (StataCorp, College Station, Texas, USA). Statistical significance level was set as $\mathrm{P}$ value 0.05 .

Using both datasets from the CHARLS (2011 baseline, 2013 follow-up), we examined the individual OOP payment for both outpatient and inpatient services among patients with CVD who reported that their last outpatient/inpatient visit was CVD-related. We then applied multilevel regression analyses to explore the association between one's financial burden (as measured by OOP payment and household CHE occurrence), and individual SES factors and rural-urban difference.

\section{RESULTS}

Prevalence of CVD and patients' demographic characteristics

Table 1 summarises the characteristics of patients with CVD in 2011 and 2013. Overall, the number of female patients was slightly higher than male patients, and around $70 \%$ of the patients with CVD were aged 55-74 years. Over $80 \%$ were living with a spouse. Most patients with CVD suffered from a co-occurring NCD, with $>80 \%$ having one or more complications. There were slightly more rural than urban patients included $(51.8 \%$ vs $48.2 \%$ in 2011 , and $53.0 \%$ vs $47.0 \%$ in 2013); furthermore, patients living in the middle part of China were slightly over-represented compared with residents of west and east China. Online supplementary table 1 presents the prevalence of CVD across different SES subgroups and the $\chi^{2}$ test results. Overall, with all covariates controlled, the difference of prevalence is more significant across education levels and occupation categories $(\mathrm{P}<0.05)$. Respondents from the education level of "vocational and above' were more likely to have CVD (19.8\% in 2011 and $22.1 \%$ in 2013), as were those from the occupation levels of 'retired/receded' (28.6\% in 2011 and $31.6 \%$ in 2013) and 'unemployed' (21.9\% in 2011 and $24.2 \%$ in 2013).

\section{OOP payment}

Regarding health service utilisation, about $15 \%$ of patients with CVD (334/2568 in 2011; 463/3056 in 2013) reported being hospitalised at least once in the past year because of CVD, and $10 \%$ of patients with CVD (260/2568 in 2011; 279/3056 in 2013) used outpatient services because of CVD at least once during the past month. From 2011 to 2013, a higher proportion of patients with CVD used inpatient service (from $13.0 \%$ to $15.1 \%$ ), and used outpatient service more frequently over 1 year (from 8.8 visits/ patient to 9.7 visits / patient).

The median number of OOP payment by service type is reported in table 2 . While the median, annual household expenditure among CHARLS respondents was 16290 Yuan (2506 USD) in 2011, and 19715 Yuan (3129 USD) in 2013 (data not shown), for patients with CVD, the total OOP payment over 12 months was 5000 Yuan (770 USD) in 2011, and 6120 Yuan (970 USD) in 2013 per capita. Thus, patients with CVD, on average, attributed $30.69 \%$ and $31.04 \%$ of expenses to OOP payment in 2011 and 2013, respectively. From 2011 to 2013, the direct 
Table 1 Demographic characteristics of the patients with CVD in CHARLS

\begin{tabular}{|c|c|c|c|c|}
\hline \multirow[b]{2}{*}{ Characteristics } & \multicolumn{2}{|c|}{$2011(n=2568)$} & \multicolumn{2}{|c|}{$2013(n=3056)$} \\
\hline & Urban & Rural & Urban & Rural \\
\hline Total $^{*}$ & 1238 (48.2) & 1330 (51.8) & 1435 (47.0) & $1621(53.0)$ \\
\hline Male & $540(43.6)$ & $548(41.2)$ & $618(43.1)$ & $672(41.5)$ \\
\hline Female & $696(51.4)$ & $781(58.8)$ & $814(56.9)$ & $944(58.5)$ \\
\hline \multicolumn{5}{|l|}{ Age (\%) } \\
\hline 45 54 years & $210(17.0)$ & $280(21.1)$ & $239(16.7)$ & 315 (19.4) \\
\hline 55 64 years & $472(38.1)$ & $488(36.7)$ & $519(36.2)$ & $543(33.5)$ \\
\hline $65 \sim 74$ years & $348(28.1)$ & 377 (28.3) & 395 (27.5) & $518(32.0)$ \\
\hline$\geq 75$ years & $208(16.8)$ & $185(13.9)$ & $282(19.7)$ & $244(15.1)$ \\
\hline \multicolumn{5}{|l|}{ Marital status (\%) } \\
\hline \multicolumn{5}{|c|}{ NCD co-occurrence (\%) } \\
\hline None & $132(10.7)$ & $160(12.0)$ & $217(15.1)$ & $242(15.0)$ \\
\hline $1 \sim 2$ kinds of NCD & $681(55.0)$ & $670(50.4)$ & $728(50.7)$ & $873(52.4)$ \\
\hline$\geq 3$ kinds of NCD & $425(34.3)$ & $500(37.6)$ & $490(34.1)$ & $506(32.6)$ \\
\hline \multicolumn{5}{|l|}{ Regional location (\%) } \\
\hline East & 365 (29.5) & $393(29.5)$ & $436(30.4)$ & $483(29.8)$ \\
\hline Middle & $488(39.4)$ & $461(34.7)$ & $564(39.3)$ & $563(34.7)$ \\
\hline West & $385(31.1)$ & $476(35.8)$ & 435 (30.3) & 575 (35.5) \\
\hline
\end{tabular}

*Numbers across the subgroups of some certain characteristics do not add up to the total because of missing values.

CHARLS, China Health and Retirement Longitudinal Survey; CVD, cardiovascular disease; NCD, non-communicable disease.

outpatient cost for a single visit decreased (by $5 \%$ ) from 400 Yuan/visit (62 USD) to 380 Yuan/visit (58 USD) per capita, but the direct inpatient cost for a single visit increased (by 22\%) from 2050 Yuan/visit (325 USD) to 2500 Yuan/visit (397 USD) per capita. Even though the cost for a single outpatient visit was lower than that of a single inpatient visit, patients with CVD spent more on outpatient services over 1 year due to higher visit frequencies, higher deductibles and lower deductions. In both 2011 and 2013, the annual burden for outpatient care (around 5930 Yuan; 912 USD) was almost twice as much as that for inpatient care (around 3350 Yuan; 515 USD), and such a difference could be found in both urban and rural patients. The results also showed that urban patients spent more on direct cost than their rural counterparts (77 Yuan/12 USD more on single inpatient visit, and 200 Yuan/31 USD more on single outpatient visit), but less on indirect cost such as transportation and accommodation. Regarding the indirect cost, for outpatient services in 2011, rural patients spent $>10$ Yuan/1.5 USD for a single visit, but urban patients spent $<5$ Yuan/0.8 USD (such a gap disappeared in 2013); for inpatient service, rural patients spent as much as 200 Yuan/31 USD for a single visit while urban patients spent 0 (such a gap consisted in 2011 and 2013). According to the third quartile of indirect cost for last inpatient hospitalisation, both urban and rural patients spent as much as 500-600 Yuan/77-92 USD.

According to the multilevel linear regression analysis (table 3), results indicate that at individual level, the amount of OOP payments increased significantly with the household annual living expenditure increase. In 2011, patients with CVD from the wealthiest (top 20\%) households spent $85 \%$ more on OOP payments as patients from the poorest (lowest 20\%) households (coefficient $=0.852$, $\mathrm{P}<0.05$ ), while the patients with CVD from the wealthier (higher 20\%) households spent $74 \%$ more as compared with the poorest (coefficient $=0.737, \mathrm{P}<0.05)$. In 2013, the difference increased to $217 \%(\mathrm{P}<0.05)$. Education level, occupation type and rural-urban difference was not found to be significantly associated with the amount of OOP payment among patients with CVD.

\section{Catastrophic health expenditures}

We further measured the incidence of catastrophic payments for healthcare in the households with at least one patient with CVD. In 2011, 44.2\% of all interviewed CVD households encountered CHE $(38.7 \%$ in urban households and $49.1 \%$ in rural households). In 2013, $48.1 \%$ of the CVD households encountered CHE, but the gap between urban $(45.2 \%)$ and rural $(50.6 \%)$ narrowed down to $5 \%$ points (data not shown). 


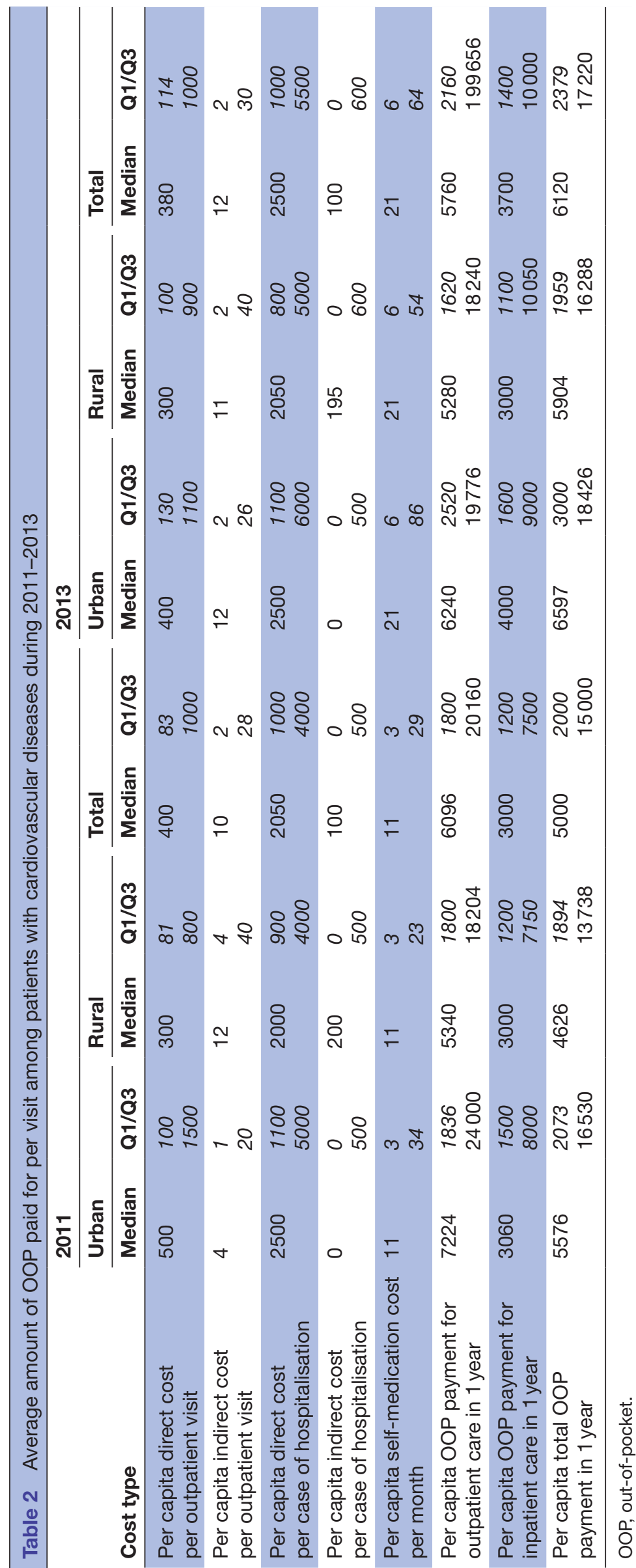


Table 3 Association between per capita OOP payment ${ }^{\dagger}$ in 1 year and socioeconomic factors among patients with CVD during 2011-2013 (multilevel linear regression ${ }^{\ddagger}$ )

\begin{tabular}{|c|c|c|c|c|c|c|}
\hline \multirow[b]{2}{*}{ SES indicator } & \multicolumn{3}{|l|}{2011} & \multicolumn{3}{|l|}{2013} \\
\hline & Coefficient & SE & $P$ value & Coefficient & SE & $P$ value \\
\hline \multicolumn{7}{|l|}{ Education (ref: primary school) } \\
\hline Middle school & -0.248 & 0.267 & 0.353 & -0.193 & 0.236 & 0.414 \\
\hline High school & 0.047 & 0.373 & 0.900 & -0.477 & 0.399 & 0.233 \\
\hline Vocational school and above & 0.370 & 0.396 & 0.349 & -0.619 & 0.426 & 0.146 \\
\hline \multicolumn{7}{|l|}{ Occupation (ref: agricultural work) } \\
\hline Employed & 0.277 & 0.326 & 0.395 & -0.592 & 0.506 & 0.242 \\
\hline Self-employed & 0.198 & 0.530 & 0.709 & -0.436 & 0.473 & 0.357 \\
\hline Retired/receded & 0.209 & 0.282 & 0.458 & -0.179 & 0.310 & 0.563 \\
\hline Unemployed & 0.253 & 0.243 & 0.298 & 0.124 & 0.174 & 0.476 \\
\hline \multicolumn{7}{|c|}{ Household expenditure (ref: the lowest $20 \%$ ) } \\
\hline Lower $20 \%$ & 0.122 & 0.283 & 0.667 & 0.384 & 0.272 & 0.158 \\
\hline Middle 20\% & 0.388 & 0.296 & 0.190 & 0.913 & 0.273 & $0.001^{*}$ \\
\hline Higher $20 \%$ & 0.737 & 0.320 & $0.021^{*}$ & 0.603 & 0.278 & $0.030^{*}$ \\
\hline Highest 20\% & 0.852 & 0.331 & $0.010^{*}$ & 1.168 & 0.263 & $<0.001^{*}$ \\
\hline \multicolumn{7}{|l|}{ Urban/rural (ref: rural) } \\
\hline Urban & -0.053 & 0.226 & 0.814 & 0.049 & 0.263 & 0.852 \\
\hline \multicolumn{7}{|l|}{ Random effect } \\
\hline SD/SE & $0.565 / 0.457$ & & & $<0.001 / 0.002$ & & \\
\hline
\end{tabular}

${ }^{*} \mathrm{P}<0.05$.

†Per capita annual OOP was log-transformed to normalise its distribution.

$\ddagger$ Variables adjusted in the model include: (1) individual level: age, sex, marital status, NCD co-occurrence and (2) community level: population size, per capita annual net income, regional location (east/middle/west part of China), availability of healthcare services and transportation condition.

CVD, cardiovascular disease; NCD, non-communicable disease; OOP, out-of-pocket; SES, socioeconomic status.

In the following multilevel logistic regression model, we explored how SES indicators were associated with the patient's household encountering catastrophic health spending (table 4). Occupation, household living expenditure level and urban-rural difference were all found to be significantly associated with the odds of encountering CHE $(\mathrm{P}<0.05)$. Regarding urban-rural difference, the odds of encountering CHE for the families from urban communities were $39 \%$ lower $(\mathrm{OR}=0.61,95 \%$ CI 0.47 to 0.80 ) than their rural counterparts in 2011; although in contrast to the analysis results in 2013, there was no relation between any community-level variables and CHE occurrence. At individual level, the odds of CHE were higher among the respondents who were unemployed than those who did agricultural work, both in 2011 (OR=1.42, 95\% CI 1.13 to 1.79$)$ and in $2013(\mathrm{OR}=1.30,95 \%$ CI 1.06 to 1.60). Compared with the patients from poorest households (ie, the lowest $20 \%$ of annual living expenditure), the odds of encountering CHE were significantly lower among patients from more wealthy households. The OR for each household group from the richest to the poorer is 0.42 (95\% CI 0.47 to 0.80$), 0.55$ (95\% CI 0.42 to 0.73 ), 0.71 (95\% CI 0.54 to 0.93 ), 1.05 (95\% CI 0.81 to 1.37 ), respectively. In addition, a descending trend of odds of encountering CHE in families of patients with CVD could be observed with the increase of household living expenditure level, although the $95 \%$ CIs were overlapped.

\section{DISCUSSION}

This study used the CHARLS data set to provide evidence on the average OOP payment and occurrence of CHE among patients with CVD. Furthermore, we explored how OOP payment and CHE were associated with individual SES and community urban-rural difference. Findings show that from 2011 to 2013, the average annual OOP payment for each patient with CVD increased from 5000 RMB to 6120 RMB (770 USD to 970 USD), while the occurrence rate of CHE in both urban and rural CVD households increased from $44.2 \%$ to $48.1 \%$. Relying heavily on outpatient care, patients with CVD spent almost twice on outpatient service as on inpatient service. Individual-level SES indicators including household living expenditure level and occupation type were found to be significantly associated with OOP payment and CHE occurrence. The urban-rural difference was significantly associated with CHE occurrence in the 2011 data. These findings underline the importance of considering individual SES and 
Table 4 OR of the occurrence of catastrophic health expenditure for each level of the socioeconomic status (SES) to the lowest group during 2011-2013 (multilevel logistic regression ${ }^{\ddagger}$ )

\begin{tabular}{|c|c|c|}
\hline & 2011 & 2013 \\
\hline SES indicator & OR $(95 \% \mathrm{Cl})$ & OR $(95 \% \mathrm{Cl})$ \\
\hline \multicolumn{3}{|l|}{$\begin{array}{l}\text { Education (ref: } \\
\text { primary school) }\end{array}$} \\
\hline Middle school & $0.99(0.78$ to 1.26$)$ & 1.07 (0.86 to 1.33$)$ \\
\hline High school & $0.95(0.65$ to 1.38$)$ & 1.15 (0.83 to 1.58$)$ \\
\hline $\begin{array}{l}\text { Vocational school } \\
\text { and above }\end{array}$ & $0.94(0.64$ to 1.40$)$ & 0.84 (0.59 to 1.19$)$ \\
\hline \multicolumn{3}{|c|}{ Occupation (ref: agricultural activities) } \\
\hline Employed & 1.07 (0.75 to 1.52$)$ & 1.02 (0.74 to 1.42$)$ \\
\hline Self-employed & $0.75(0.48$ to 1.18$)$ & 0.76 (0.54 to 1.08$)$ \\
\hline Retired/receded & $1.05(0.76$ to 1.46$)$ & $1.34(1.00$ to 1.78$)$ \\
\hline Unemployed & $1.42(1.13 \text { to } 1.79)^{*}$ & $1.30(1.06 \text { to } 1.60)^{\star}$ \\
\hline \multicolumn{3}{|c|}{ Household expenditure (ref: the lowest $20 \%$ ) } \\
\hline Lower 20\% & 1.05 (0.81 to 1.37$)$ & $0.73(0.57 \text { to } 0.94)^{*}$ \\
\hline Middle $20 \%$ & $0.71(0.54 \text { to } 0.93)^{*}$ & $0.59(0.46 \text { to } 0.76)^{\star}$ \\
\hline Higher $20 \%$ & $0.55(0.42 \text { to } 0.73)^{\star}$ & $0.53(0.41 \text { to } 0.68)^{\star}$ \\
\hline Highest $20 \%$ & $0.42(0.31 \text { to } 0.56)^{\star}$ & $0.43(0.33 \text { to } 0.55)^{\star}$ \\
\hline \multicolumn{3}{|l|}{$\begin{array}{l}\text { Urban/rural (ref: } \\
\text { rural) }\end{array}$} \\
\hline Urban & $0.61(0.47 \text { to } 0.80)^{*}$ & 0.86 (0.68 to 1.09$)$ \\
\hline \multicolumn{3}{|l|}{ Random effect } \\
\hline SD† & 0.472 & 0.405 \\
\hline
\end{tabular}

${ }^{*} \mathrm{P}<0.05$.

†Stata only reports SD for multilevel logistic regression. $¥$ Variables adjusted in the model include: (1) individual level: age, sex, marital status, NCD co-occurrence and (2) community level: population size, per capita annual net income, regional location (east/middle/west part of China), availability of healthcare services and transportation condition.

urban-rural difference when designing health cost-control strategies, and emphasise the need to understand and examine variation in health expenditure among different patient groups with CVD.

Although we used nationally representative household survey data to estimate health expenditure among patients with CVD and show an association with SES, our study also had some limitations. First, we estimated the average OOP payment of patients with CVD over 12 months who self-reported that their last medical visit was for a CVD. It is possible that additional patients with CVD were not included (because their last medical visit was not CVD-related), thus leading to an underestimation in actual CVD-related costs for patients. It is also possible some patients visited the doctor for co-occurring diseases, which could lead to an overestimation of cost. Second, the natural ageing process of the followed-up patients, the price fluctuation in health cost, along with currency inflation could potentially have influenced patients' healthcare cost between 2011 and 2013. Third, given that, at the time of analysis, CHARLS had only released its full 2011 and 2013 survey data (part of the 2015 follow-up data has been released in 2017), we only captured the data in 2 years and were therefore unable to evaluate the long-term effects of the current medical insurance scheme. Since CHARLS is a household survey and all cost variables were self-reported data, a further parallel study using health administrative databases could help to better understand the changing trend of OOP payment among patients with CVD with different SES features.

Compared with the two other studies conducted in China that estimated the direct cost of patients with CVD, our estimations of the annual medical cost were in the middle: Wang et al reported the average cost per CVD hospitalisation in 2012 was 2236.29 USD (around 14700 RMB) in Shanghai alone ${ }^{25}$; Liu et al estimated the annual financial burden of patients with CVD in urban areas was 8569.3 RMB (1320 USD) using a nationwide household survey conducted between 2007 and 2011. ${ }^{26}$ Such differences could be influenced by the price factor as well as the sampling areas. As mentioned earlier, only those whose reason for their last medical visit was CVD were included. This could lead to us having underestimated individual annual cost while also potentially missing some genuine patients with CVD. Furthermore, unlike the aforementioned papers, our analysis used national data from both urban and rural areas, which could impact the estimated direct cost, relative to other studies. The readers should thus be cautious when comparing the present results directly to other studies.

An unexpected finding from our study is that annual cost for outpatient service is higher than annual cost for inpatient service among these patients with CVD. Normally, the relatively high cost for a CVD inpatient service, like coronary artery bypass or cerebral artery bypass, would incur higher OOP payment for CVD inpatient service than for outpatient services. Our results could be explained by the fact that patients with CVD had much higher utilisation of outpatient service (as compared with inpatient services), higher deductibles and copayment rate and a lower maximum reimbursement limit. To date, some insurance schemes do not cover outpatient services in certain provinces. In general, the Chinese basic medical insurance schemes do not address the demand for frequent outpatient visits among patients with chronic conditions (Yip and Hsiao, 2009)..$^{8}$

Previous studies have reported that patients from wealthier households have more access to health services, and are more likely to both use the services and stick with treatment plans because financial hardship is not a barrier to this group. ${ }^{27-29}$ Our study similarly found that patients with CVD with higher SES levels were more likely to spend more on health services and medication, and had lower odds of encountering catastrophic spending when compared with the lowest level. It was a reasonable conjecture that the poor have limited access to healthcare services as well as a higher tendency to not visit a doctor because of financial hardships when encountering 
chronic illness. ${ }^{30}$ Jian $e t$ al also found that rural Chinese people with chronic diseases were two times more likely to drop out of treatment because of financial difficulties than those from urban areas. ${ }^{31}$ In this study, we also found that rural patients were less likely to use inpatient services compared with urban patients, but no significant difference was observed regarding outpatient care. On one hand, due to the fact that health resources tend to gather in economically developed areas in China, it is hard for patients living in rural and remote areas to get access to appropriate health services. ${ }^{32}$ On the other hand, patients with a higher economic status in China have higher payment capacity, and hence, they are more likely to use health services when in need. ${ }^{15}$ Indeed, although there is more governmental subsidisation directed towards patients with CVD who need inpatient services, the utilisation among the rural patients is lacking. This defeats the goal of the basic medical insurance introduced by the Chinese government.

Although the existing inequality of disease burden across different SES groups is acknowledged, the Chinese medical insurance system is still somewhat pro-rich. Our findings show that, with regard to inpatient service costs only, patients from the poorest households had the highest actual copayment rate of $70 \%$, whereas the wealthiest had only $56 \%$. Besides direct medical costs, people living in lower SES communities, which in most cases also lack advanced medical centres, have to spend more money on transportation or accommodation in order to receive appropriate healthcare. ${ }^{18}$ In our study, rural patients spent 200 Yuan (30 USD) more than their rural counterparts on indirect cost to receive inpatient service. Thus, policymakers should take indirect medical costs into consideration when re-allocating health resources, and work towards lowering copayment rates among the poor.

One positive finding from this study is that the different odds of encountering CHE between urban and rural CVD households was only significant in 2011, but not in 2013. This could be in part due to the efforts of the 2009 Chinese health reform in China, which invested resources to strengthen the primary healthcare system (eg, increasing community health centres and village clinics). It also might be due to the process of merging the two basic medical insurance schemes, URBMI and NRCMI. This is currently being piloted in several cities. The aim of this merge is to provide identical insurance schemes to both urban unemployed residents and rural residents. It should be pointed out that although almost all the provinces adopt the structure of three basic insurance schemes, the benefit design regarding copayment rate, deductibles and covered services might vary greatly across provinces and even cities in one province. Citizens from richer provinces normally have better insurance schemes, as well as more accessible and higher quality of health services. This is partly due to the political development and enactment of medical insurance schemes. Briefly, the Chinese Central Government devises general national health policies and guidance documents, then the individual province government enacts its own specific health policies and guidance (eg, copayment rate, annual maximum limit). Thus, the healthcare benefits may vary by province. Future studies should build on our work by doing a cross-comparison of provincial policies and its association with CVD inpatient and outpatient services by employment, economic and education status.

Although the results from this study could not be generalisable to the general patient population in China, neither could it represent patients with CVD as a whole, our results of cost analysis and its association with patients' SES reveal an unfortunate fact: the current health system in China still has health inequity across different incomegroups for patients with chronic diseases like CVD. The medical insurance scheme structure hinders the poor from obtaining adequate health services, while increasing the likelihood of financial burden due to disproportionate health expenditure. ${ }^{33}$ Reasons behind the insufficient implementation of such national policies might include lack of health professionals in primary care centres and in rural areas, relatively shallow benefit design of the three basic medical insurance schemes and incomplete transformation from the hospital-centred system to a primary care-based delivery system. ${ }^{34}$ Much is still to be desired with regard to our understanding of how to effectively alleviate medical financial risk for the poor. We implore that more research be conducted in this area.

\section{CONCLUSION}

In conclusion, through using nationally representative data from CHARLS, this study has provided the cost estimation for patients with CVD and investigated whether, and to what extent, an individual's SES level and community conditions were associated with health expenditure. The findings suggest that wealthier people spend more money on the health services but are less likely to encounter CHE. Accumulatively, patients with CVD have higher financial burden for outpatient service (which they use overwhelmingly more) as compared with that for inpatient service. The medical insurance scheme should be more financially balanced between outpatient and inpatient care, which could provide better financial risk protection to patients with chronic conditions. There is a need for Chinese policymakers to alter current pro-rich medical insurance policies to ensure that the poor can access essential health services without having to worry about incurring or worsening financial hardship.

Contributors $\mathrm{CL}$ analysed and interpreted the data and wrote the manuscript. B-RY wrote portions of the manuscript and contributed to its revisions. WJ helped on the study design and revised the manuscript.

Funding This work was supported by the National Natural Science Foundation of China, Grant No. 71774003.

\section{Competing interests None declared.}

Patient consent Detail has been removed from this case description/these case descriptions to ensure anonymity. The editors and reviewers have seen the detailed information available and are satisfied that the information backs up the case the authors are making. 
Ethics approval CHARLS was approved by the Ethical Review Committee (IRB) at Peking University, Beijing, China.

Provenance and peer review Not commissioned; externally peer reviewed.

Data sharing statement Technical appendix, statistical code and dataset available from the CHARLS repository, http://charls.pku.edu.cn.

Open Access This is an Open Access article distributed in accordance with the Creative Commons Attribution Non Commercial (CC BY-NC 4.0) license, which permits others to distribute, remix, adapt, build upon this work non-commercially, and license their derivative works on different terms, provided the original work is properly cited and the use is non-commercial. See: http://creativecommons.org/ licenses/by-nc/4.0/

(c) Article author(s) (or their employer(s) unless otherwise stated in the text of the article) 2018. All rights reserved. No commercial use is permitted unless otherwise expressly granted.

\section{REFERENCES}

1. World Health Organization. Cardiovascular diseases (CVDs). http:// www.who.int/mediacentre/factsheets/fs317/en/ (assessed on Nov 24 2017).

2. Schnall PL, Dobson M, Globalization LP. Work, and Cardiovascular Disease. Int J Health Serv 2016;46:656-92.

3. Williams DR, Costa MV, Odunlami AO, et al. Moving upstream: how interventions that address the social determinants of health can improve health and reduce disparities. J Public Health Manag Pract 2008;14:S8-17.

4. Macintyre S, Ellaway A, Cummins S. Place effects on health: how can we conceptualise operationalise and measure them?. Soc Sci Med 2002;55:125-39.

5. Lantz PM, Lynch JW, House JS, et al. Socioeconomic disparities in health change in a longitudinal study of US adults: the role of healthrisk behaviors. Soc Sci Med 2001;53:29-40.

6. Yang L, Wu M, Cui B, et al. Economic burden of cardiovascular diseases in China. Expert Rev Pharmacoecon Outcomes Res 2008;8:349-56.

7. National Center for Cardiovascular Diseases of China. Report on Cardiovascular Diseases in China (2016). Beijing, China: Encyclopedia of China Publishing House, 2017.

8. The Central Committee of the Communist Party of China, the State Council of China. Opinions of the CPC Central Committee and the State Council on deepening the health care system reform. http:// www.gov.cn/test/2009-04/08/content_1280069.htm (accessed 20 May 2017).

9. Yu H. Universal health insurance coverage for 1.3 billion people: What accounts for China's success? Health Policy 2015;119:1145-52

10. WHO Representative Office China: Health Sector Reform in China. http://www.wpro.who.int/china/mediacentre/factsheets/health_ sector_reform/en. (assessed on 20 May 2017).

11. Meng $\mathrm{Q}$, Fang $\mathrm{H}$, Liu $\mathrm{X}$, et al. Consolidating the social health insurance schemes in China: towards an equitable and efficient health system. Lancet 2015;386:1484-92.

12. Sun J, Deng S, Xiong $X$, et al. Equity in access to healthcare among the urban elderly in China: does health insurance matter? Int $J$ Health Plann Manage 2014;29:e127-e144.

13. Yip WC, Hsiao WC, Chen W, et al. Early appraisal of China's huge and complex health-care reforms. Lancet 2012;379:833-42.
14. Yip W, Hsiao WC. Non-evidence-based policy: how effective is China's new cooperative medical scheme in reducing medical impoverishment? Soc Sci Med 2009;68:201-9.

15. Guo N, Iversen T, Lu M, et al. Does the new cooperative medical scheme reduce inequality in catastrophic health expenditure in rural China? BMC Health Serv Res 2016;16:653.

16. Mchenga M, Chirwa GC, Chiwaula LS. Impoverishing effects of catastrophic health expenditures in Malawi. Int $J$ Equity Health 2017;16:25

17. Barasa EW, Maina T, Ravishankar N. Assessing the impoverishing effects, and factors associated with the incidence of catastrophic health care payments in Kenya. Int J Equity Health 2017:16:31.

18. Rout HS. Socio-economic Factors and Household Health Expenditure. J Health Manag 2008;10:101-18.

19. Evans TG, Kieny MP. Systems science for universal health coverage. Bull World Health Organ 2017;95:484.

20. Zhao Y, Hu Y, Smith JP, et al. Cohort profile: the China Health and Retirement Longitudinal Study (CHARLS). Int J Epidemiol 2014;43:61-8.

21. O'Donnell O, Van Doorslaer E, Wagstaff A, et al. Analyzing Health Equity Using Household Survey Data. https://openknowledge. worldbank.org/handle/10986/6896 (accessed 20 May 2017).

22. Xu K, Evans DB, Kawabata K, et al. Household catastrophic health expenditure: a multicountry analysis. Lancet 2003;362:111-7.

23. Li YN, Nong DX, Wei B, et al. The impact of predisposing, enabling, and need factors in utilization of health services among rural residents in Guangxi, China. BMC Health Serv Res 2016;16:592.

24. Pradhan J, Dwivedi R. Do we provide affordable, accessible and administrable health care? An assessment of SES differential in out of pocket expenditure on delivery care in India. Sex Reprod Healthc 2017:11:69-78.

25. Wang S, Petzold M, Cao J, et al. Direct medical costs of hospitalizations for cardiovascular diseases in Shanghai, China: trends and projections. Medicine 2015;94:e837.

26. Liu M, Sun LH, Liu G. [Economic burden and economic risk of five major chronic diseases among Chinese urban residents]. Beijing Da Xue Xue Bao Yi Xue Ban 2014;46:782-9.

27. Chen $Y$, Li L, Zhang Q, et al. Use of drug treatment for secondary prevention of cardiovascular disease in urban and rural communities of China: China Kadoorie Biobank Study of 0.5 million people. Int J Cardiol 2014;172:88-95.

28. Mukherjee S, Haddad S, Narayana D. Social class related inequalities in household health expenditure and economic burden: evidence from Kerala, south India. Int J Equity Health 2011;10:1.

29. Zhang C, Lei X, Strauss J, et al. Health Insurance and Health Care among the Mid-Aged and Older Chinese: Evidence from the National Baseline Survey of CHARLS. Health Econ 2017;26:431-49.

30. Zhao X, Ming DY, Wj M. Utilization and cost of outpatient care and their influencing factors among middle and aged peasant-workers in China. Beijing Da Xue Xue Bao 2015;47:464-8.

31. Jian W, Chan KY, Reidpath DD, et al. China's rural-urban care gap shrank for chronic disease patients, but inequities persist. Health Aff 2010;29:2189-96.

32. Shen S, Hou X. The integration goal of the fragmented social medical insurance system in China (in Chinese). Social Sciences in Guangdong 2012;3:19-25

33. Ma J, Xu J, Zhang Z, et al. New cooperative medical scheme decreased financial burden but expanded the gap of income-related inequity: evidence from three provinces in rural China. Int $J$ Equity Health 2016;15:72.

34. Yip W, Hsiao W. Harnessing the privatisation of China's fragmented health-care delivery. Lancet 2014;384:805-18. 